\title{
Relation between colorimetric colour-differences and perceived ones for saturated colour chips with constant value and chroma
}

\author{
Koichi IKEDA*, Masaharu NAKAYAMA* \\ and Kiyoshige OBARA*
}

Perceived colour-differences of colour chips are estimated subjectively by means of ratio estimation method under standard illuminant $A$ and daylight $D_{65}$, and are compared with their colorimetric ones in the CIE 1976 $L^{*} u^{*} v^{*}$, the CIE $1976 L^{*} a^{*} b^{*}$, a modified $L^{*} u^{*} v^{*}$, and a modified Cube-root colour spaces.

The set of colour chips used in the experiment consists of one hundred and sixty hues with constant value and chroma of $6 / 8$ in the Munsell renotation system. Colorimetric colour-differences of the pairs of colour chips are within the range of 0.89 to 17.56 under daylight illuminant $D_{65}$ in the CIE $1976 L^{*} a^{*} b^{*}$ uniform colour space.

Results obtained are summarized as follows:

(1) Relative values of perceived colour-differences change little even when the illuminant changes from $D_{65}$ to $A$, and the constancy of perceived colour-difference holds as well as in the case of colour chips of lower chroma.

(2) Under the daylight $D_{65}$, the degrees of correlation between perceived colour-differences and colorimetric ones are little different among the colour spaces examined, but under the illuminant $A$ the degree in the CIE $L^{*} u^{*} v^{*}$ colour space is a little lower than the others.

(3) The variation with hue angle of the ratio of colorimetric colourdifferences under the illuminant $A$ and those under the illuminant $D_{65}$ in the CIE $L^{*} u^{*} v^{*}$ colour space is the largest, and that in the Cube-root colour space is the smallest.

(4) The correction to the chromatic adaptation in terms of colour-differences is well done in the Cube-root colour space, and it is scarcely done in the CIE $L^{*} u^{*} v^{*}$ colour space.

\section{Introduction}

As spaces for specifing colours, the $x, y$ coordinates system and the Munsell renotation system are today widely in use. These systems have already proven to be convenient for individually specifing the characteristics of the psychophysical aspects of colours and those of the perceived aspects, respectively. They, however, are not suitable for estimating the appearance of colours and colourdifferences under varied conditions of observation. Various uniform colour spaces have hitherto been investigated in an attempt to correlate between psychophysical aspects and perceived ones, and two types of uniform colour spaces, i.e., the CIE 1976 $L^{*} u^{*} v^{*}$ and the CIE $1976 L^{*} a^{*} b^{*}$, and the corresponding colour-difference formulae in these spaces are at present recommended by the $\mathrm{CIE}^{1}$.

These CIE recommendations are, however, of a provisional nature. The CIE therefore suggests and emphasizes the need for developing a more thoroughgoing research with the guidelines re.

* Faculty of Science and Technology, Tokyo Rika Daigaku commended by the CIE ${ }^{2)}$ for the study of quantitative estimation of colour-differences.

For the purpose of estimating the variations of perceived colour-differences caused by the change of illuminant, the authors performed a colourdifference estimation experiment by means of the ratio estimation method for low chroma colour chips having Chroma 2 to 4 and Value 6 and consisting of two hundreds and twelve hues in the Munsell renotation system. This study have been reported in the previous papers ${ }^{3)}$ to 6 ).

In this study, a similar colour-difference evaluation test is conducted using colour chips having equal Value 6 and high Chroma 8 to investigate the relations between colorimetric colour-differences and perceived ones in the CIE $1976 L^{*} u^{*} v^{*}$, the CIE $1976 L^{*} a^{*} b^{*}$, a modified $L^{*} u^{*} v^{*}$ and a modified Cube-root colour spaces, and obtained results are compared with the results by low chroma colour chips.

Most of the studies made so far, both at home and abroad, were concerned mainly with the discrimination thresholds of aperture colours. Until recent time, none has really successfully attempted the colour-difference evaluation experiment using 
the ratio estimation method, in which the magnitude of colour-differences themselves is subjectively estimated. The application of this technique ap-

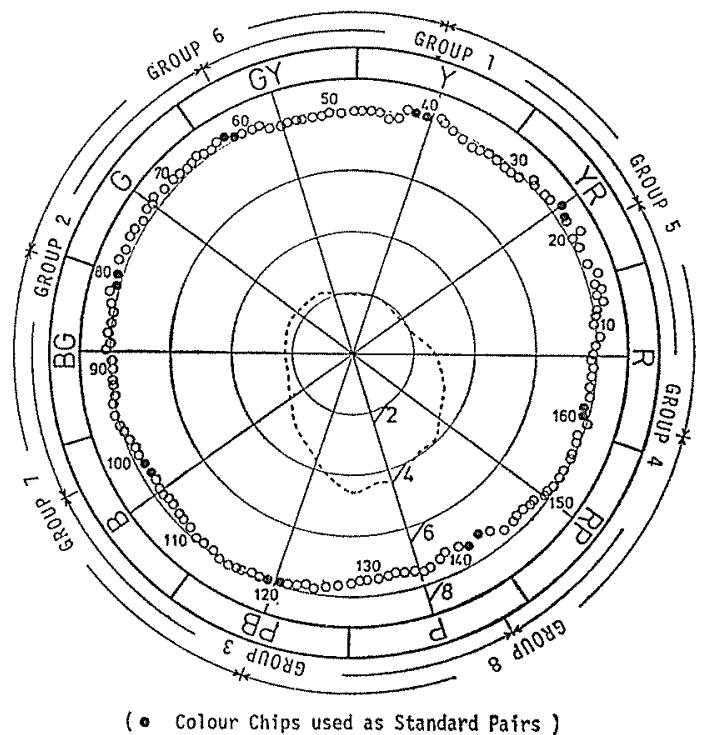

(a) Munsel1 Renotation System

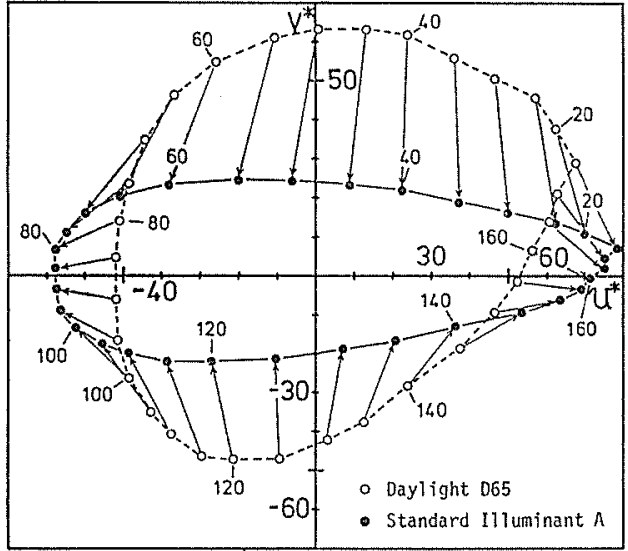

(b) CIE L*u* ${ }^{*}$ Space

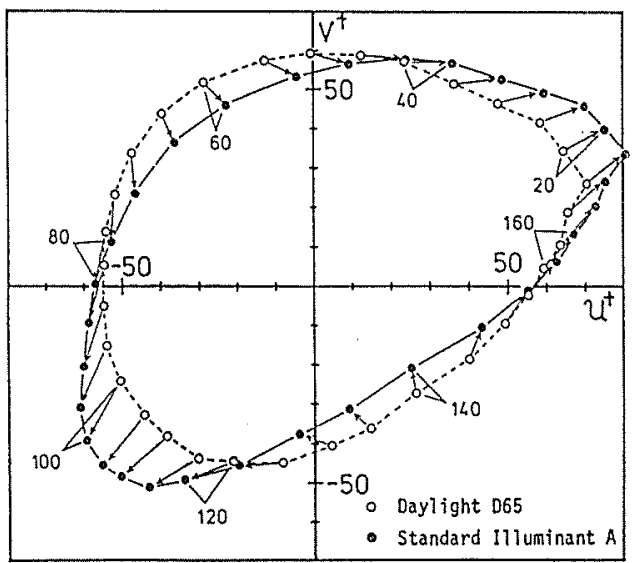

(d) L*utut Space (Modified $L^{*} u^{*} v^{*}$ Space) pears to be difficult. In actuality, however, the method can be employed in various colour-differences ranging from small colour-differences to large ones. In fact, it was confirmed that sufficiently reliable data could be obtained on the experiments of low chroma colour chips, as already described in the previous papers. Lately, a colourdifference evaluation study is being undertaken using this technique by other group ${ }^{7}$.

\section{Colour chips used for the es- timation of colour-differences}

Colour chips used for determination of colourdifferences in the visual and psychological experiment were prepared by the Japan Color Research Institute. The colour chips were so designed that hue angles could be spaced equally on the hue circle with the equal Value 6 and equal Chroma 8 in the Munsell renotation space and were Iusterfinished.

The spectral reflectances of all the colour chips were measured three times each with a spectrophotometer, and the coordinates in each colorimetric space were computed by means of a computer. The coordinates of colour chips in the

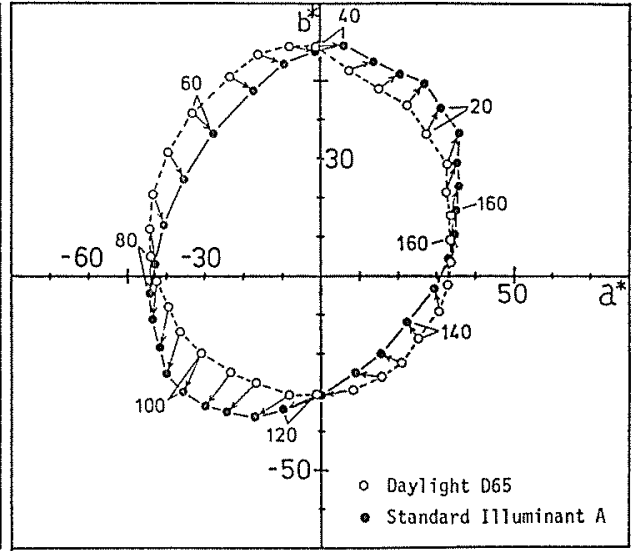

(c) CIE L*a*b* Space

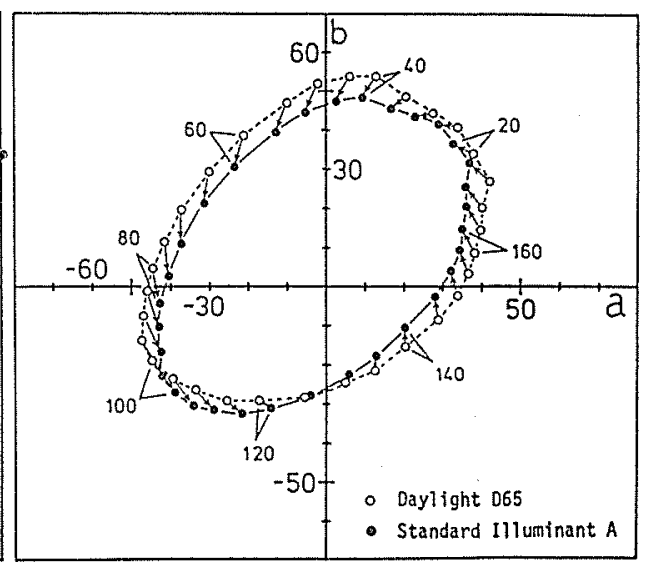

(e) Modified Cube-root Space

Fig. 1 Coordinates in each colorimetric space of colour chips used.

Only $1 / 5$ of all colour chips is shown except in the Munsell renotation system. 
Munsell renotation system, the CIE $1976 L^{*} u^{*} v^{*}$, the CIE $1976 L^{*} a^{*} b^{*}$, a modified $L^{*} u^{*} v^{*}$ and a modified Cube-root colour spaces are shown in Fig. 1.(a) through (e), respectively. The dotted line in the Munsell renotation system in Fig. 1(a) represents the coordinates of the colour chips used in the experiment on low chroma, and the group of these colour chips was designed to lie circularly in the CIE $1964 U^{*} V^{*} W^{*}$ uniform colour space. In the Munsell renotation system, therefore, the chroma changes from 2 to 4 .

Here, the colorimetric spaces are defined by:

(1) CIE $1976 L^{*} u^{*} v^{*}$ Space

$$
\begin{aligned}
& L^{*}=116\left(Y / Y_{0}\right)^{1 / 3}-16 \\
& u^{*}=13 L^{*}\left(u^{\prime}-u_{0}^{\prime}\right) \\
& v^{*}=13 L^{*}\left(v^{\prime}-v_{0}{ }^{\prime}\right)
\end{aligned}
$$

where

$$
\begin{aligned}
u^{\prime} & =4 X /(X+15 Y+3 Z) \\
v^{\prime} & =9 Y /(X+15 Y+3 Z) \\
u_{0}^{\prime} & =4 X_{0} /\left(X_{0}+15 Y_{0}+3 Z_{0}\right) \\
v_{0}^{\prime} & =9 Y_{0} /\left(X_{0}+15 Y_{0}+3 Z_{0}\right)
\end{aligned}
$$

(2) CIE $1976 L^{*} a * b *$ Space

$$
\begin{aligned}
& L^{*}=116\left(Y / Y_{0}\right)^{1 / 3}-16 \\
& a^{*}=500\left[\left(X / X_{0}\right)^{1 / 3}-\left(Y / Y_{0}\right)^{1 / 3}\right] \\
& b^{*}=200\left[\left(Y / Y_{0}\right)^{1 / 3}-\left(Z / Z_{0}\right)^{1 / 3}\right]
\end{aligned}
$$

(3) Modified $L * u^{*} v^{*}$ Space

$$
\begin{aligned}
& L^{*}=116\left(Y / Y_{0}\right)^{1 / 3}-16 \\
& u^{\dagger}=13 L^{*}\left(u^{\prime \prime}-u_{0}^{\prime \prime}\right) \\
& v \dagger=13 L^{*}\left(v^{\prime \prime}-v_{0}{ }^{\prime \prime}\right)
\end{aligned}
$$

$$
\}
$$

where

$$
\begin{aligned}
u^{\prime \prime} & =4 X_{\mathrm{c}} /\left(X_{c}+15 Y_{\mathrm{c}}+3 Z_{\mathrm{c}}\right) \\
v^{\prime \prime} & =9 X_{\mathrm{c}} /\left(X_{\mathrm{c}}+15 Y_{\mathrm{c}}+3 Z_{\mathrm{c}}\right) \\
u_{0}{ }^{\prime \prime} & =4 X_{\mathrm{c} 0} /\left(X_{\mathrm{c} 0}+15 Y_{\mathrm{c} 0}+3 Z_{c 0}\right) \\
v_{0}{ }^{\prime \prime} & =9 X_{c 0} /\left(X_{c 0}+15 Y_{c 0}+3 Z_{c 0}\right) \\
X_{\mathrm{c}} & =X / X_{0}, \quad Y_{c}=Y / Y_{0}, \quad Z_{\mathrm{c}}=Z / Z_{0}
\end{aligned}
$$

Also, against the achromatic background

$$
X_{\mathrm{c} 0}=X_{0} / X_{0},=1, \quad Y_{\mathrm{c} 0}=Y_{0} / Y_{0}=1, Z_{c 0}=Z_{0} / Z_{0}=1
$$

(4) Modified Cube-root Space

$$
\begin{aligned}
L & =25.29\left(100 G / G_{0}\right)^{1 / 3}-18.38 \\
a & =106.0\left[\left(100 R / R_{0}\right)^{1 / 3}-\left(100 G / G_{0}\right)^{1 / 3}\right] \\
b & =42.34\left[\left(100 G / G_{0}\right)^{1 / 3}-\left(100 B / B_{0}\right)^{1 / 3}\right]
\end{aligned}
$$

where

$$
\begin{aligned}
& R=1.1084 X+0.0852 Y-0.1454 Z \\
& G=-0.0010 X+1.0005 Y+0.0004 Z \\
& B=-0.0062 X+0.0394 Y+0.8192 Z \\
& R_{0}=1.1084 X_{0}+0.0852 Y_{0}-0.1454 Z_{0} \\
& G_{0}=-0.0010 X_{0}+1.0005 Y_{0}+0.0004 Z_{0} \\
& B_{0}=-0.0062 X_{0}+0.0394 Y_{0}+0.8192 Z_{0}
\end{aligned}
$$

In each space above, $X, Y$ and $Z$ represent tristimulus values of an object, and $X_{0}, Y_{0}$ and $Z_{0}$ represent those of an illuminant.

Equations (1) and (2) show the CIE 1976 uniform colour spaces, and equations (3) and (4) indicate the formulae that have been transformed to compensate the chromatic adaptation for the CIE $L^{*} u^{*} v^{*}$ space and the Cube-root space with
Reilly coefficients ${ }^{8)}$, respectively, based on AdamsNickerson's concepts.

Two sets of colour chips with 160 different hues are prepared, i.e., a total of 320 chips, in the experiment $I$. They were divided into such four: ranges of $3.0 \mathrm{YR}$ to $8.0 \mathrm{GY}, 8.0 \mathrm{GY}$ to $2.5 \mathrm{~B}, 2.5 \mathrm{~B}$ to $8.5 \mathrm{P}$, and $8.5 \mathrm{P}$ to $3.0 \mathrm{YR}$ as each range contained 40 hues. In each of these ranges, a pair of colour chips as a standard pair was prepared from those located nearly in the middle, and 39 test pairs from the remainder in random combinations for comparison purposes.

In the experiment II, colour chips were prepared for both the standard pair and the comparison pairs in the same manner as that in the experiment I, using the same remeasured colour chips, but the above ranges were changed to about $45^{\circ}$ by hueangle in the Munsell renotation space, i.e., $1.2 \mathrm{R}$ to $5.7 \mathrm{Y}, 5.7 \mathrm{Y}$ to $0.4 \mathrm{BG}, 0.4 \mathrm{BG}$ to $4.9 \mathrm{~PB}$, and $4.9 \mathrm{~PB}$ to $1.2 \mathrm{R}$.

The colorimetric colour-differences of the pairs

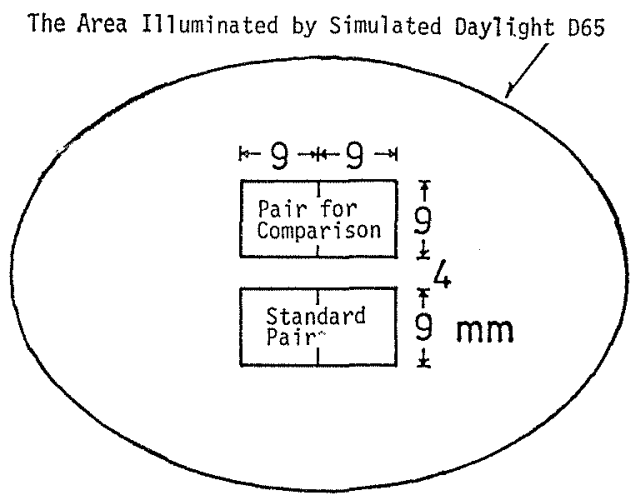

irg. 2 Dispositions of the standard pair and test pair of colour chips.

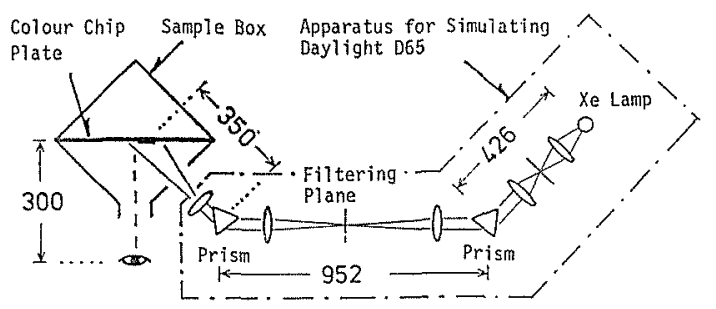

(a) Experiment under Daylight D65

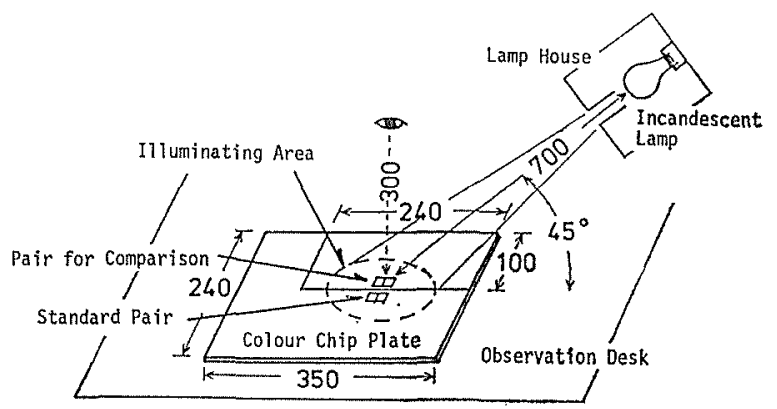

(b) Experiment under Standard Lliuminant $A$

Fig. 3 Conditions of perceived colour-differences estimation experiment. 
prepared from eight groups' colour chips under the daylight $D_{65}$ were in the range of 1.0 to 2.45 in the CIE $L^{*} a^{*} b^{*}$ space for the standard pairs and in the range of 0.89 to 17.56 in the same space for the comparison pairs. The coordinates of the colour chips used as standard pairs are shown in the Munsell renotation system of Fig. 1(a).

\section{Evaluation of colour-differen- ces by ratio estimation method}

As illuminants for illuminating the colour chips in the colour-difference experiments, the daylight illuminant $D_{65}$ and the standard illuminant $A$ were adopted. The daylight $D_{65}$ was simulated by using an apparatus for generating the illuminant with arbitiary spectral energy distribution ${ }^{9)}$, and the standard illuminant $A$ was that whose colour temperature was regulated to $2856 \mathrm{~K}$ by a semiwhite incandescent lamp $(100 \mathrm{~V}, 100 \mathrm{~W})$ lighted at about $115 \mathrm{~V}$.

Observation conditions were:

(1) $45^{\circ} /$ normal observation at about $30 \mathrm{~cm}$ distance from the pairs of colour chips.

(2) The luminance of a white paper "Kent" set on the plane of the colour chips was $50 \mathrm{~cd} / \mathrm{m}^{2}$ under the daylight $D_{65}$ and $100 \mathrm{~cd} / \mathrm{m}^{2}$ under the standard illuminant $\mathrm{A}$.

(3) The field of illumination was limited, but the visual angle subtended by the illuminated area was more than $10^{\circ}$, and more than twenty-five times as large as the area of the test colour chip.

As shown in Fig. 2, each pair of colour chips consisted of two square colour chips with their
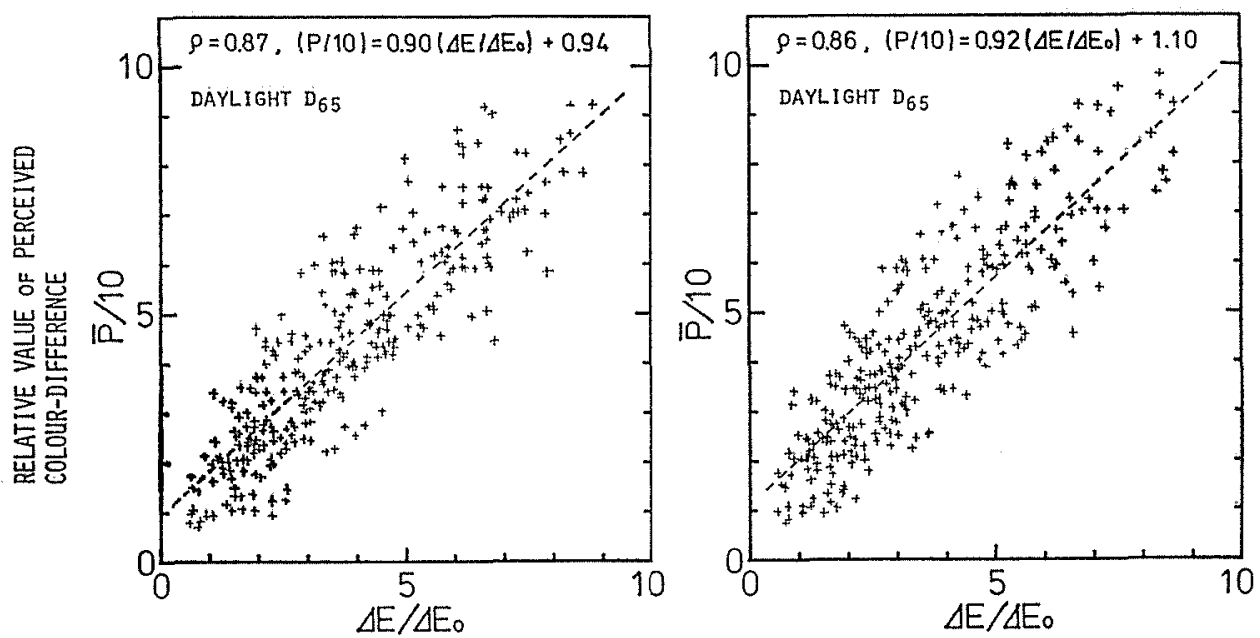

RELATIVE VALUE OF COLORIMETRIC COLOUR-DIFFERENCE

(a) CIE L* $u^{*} v^{*}$ Space

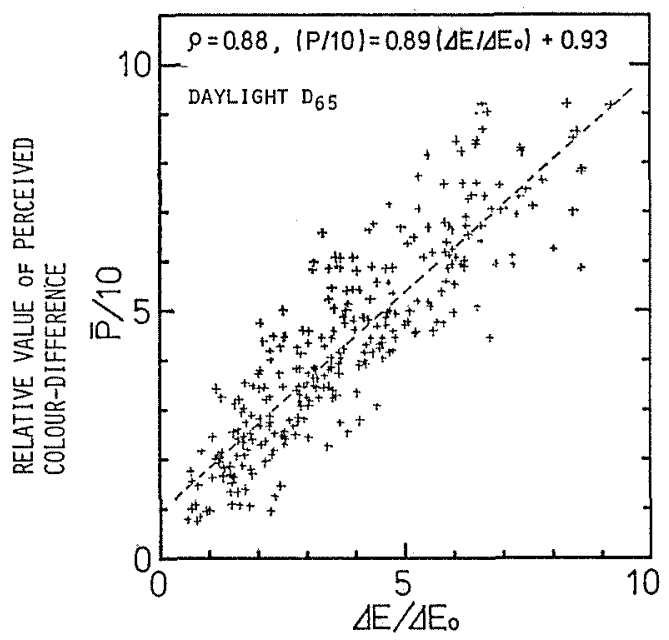

(b) CIE L*a*b* Space

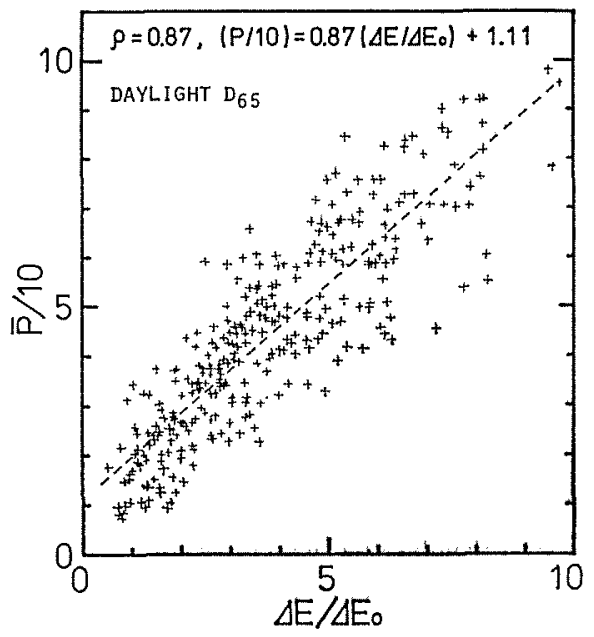

RELATIVE VALUE OF COLORIMETRIC COLOUR-DIFFERENCE (d) Modified Cube-root Space

Fig. 4 Correlation diagrams of the relative values of perceived colour-differences under the daylight $D_{65}$ to those of colorimetric ones. 
each side $9 \mathrm{~mm}$ long placed in contact with each other. The exchangeable test pair for comparison was set $4 \mathrm{~mm}$ away from the fixed standard pair, and those pairs was simultaneously displayed against an achromatic background with a visual reflectance of $\mathrm{Y} \simeq 40$. Each colour chip subtends about $2.4^{\circ}$ of visual angle.

For evaluations of colour-differences, the ratio estimation technique, which the ratios of the perceived colour-differences of the comparison pairs to the difference of the standard pair were subjectively determined by observers, was employed. Observers were requested to evaluate the relative value of perceived colour-difference of the test pair to that of the standard pair, and then answered the number multiplied it by ten.

Two male university students aged 21 to 24 , with normal colour vision, were employed as observers for each experiment. Prior to the formal experiment, the observers were fully trained to properly evaluate colour-differences by the ratio estimation method.

The observers started on the judgements of perceived colour-differences of the test pairs after they had adapted to the illuminant for a period of fifteen minutes, and the time needed to judge the thirty-nine test pairs in one sequence was twenty to twenty-five minutes for each observer. Each observer repeated this procedure eight times in the experiment I and fifteen times in the experiment II per one colour chips' group, and the order of the test pairs was changed in a random order to each observer and each experiment. To evaluated the average $(\bar{P})$ of the judged values of
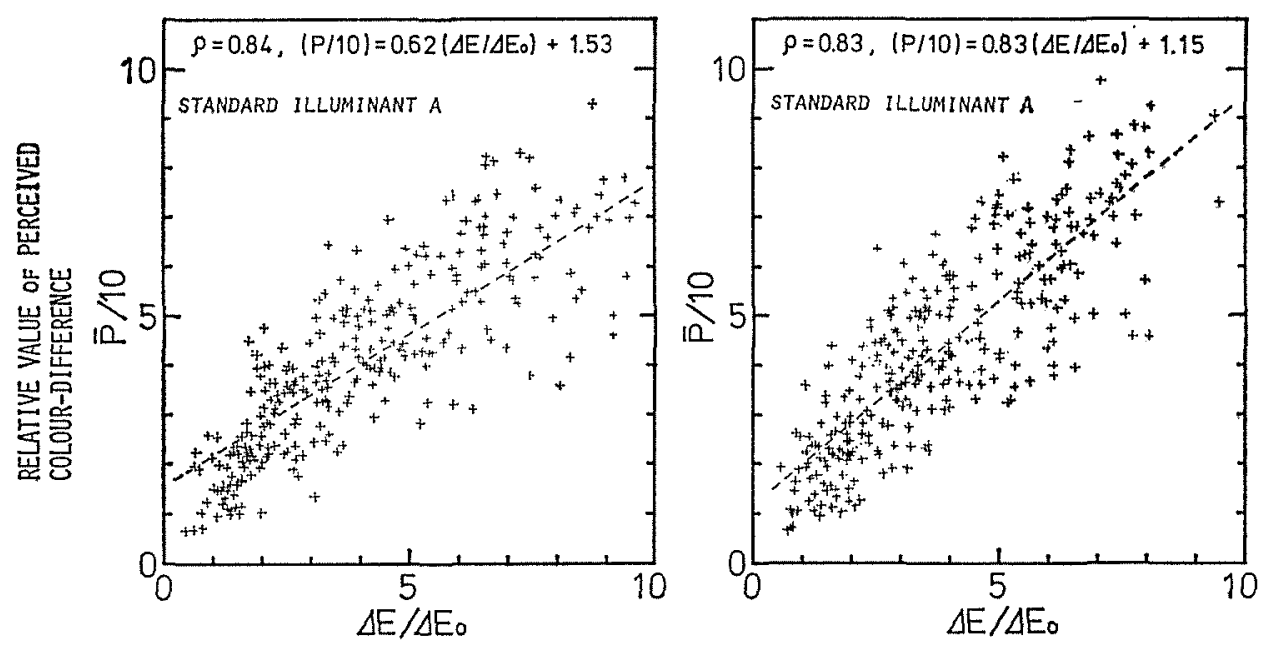

RELATIVE VALUE OF COLORIMETRIC COLOUR-DIFFERENCE

(a) CIE L* $u^{*} V^{*}$ Space

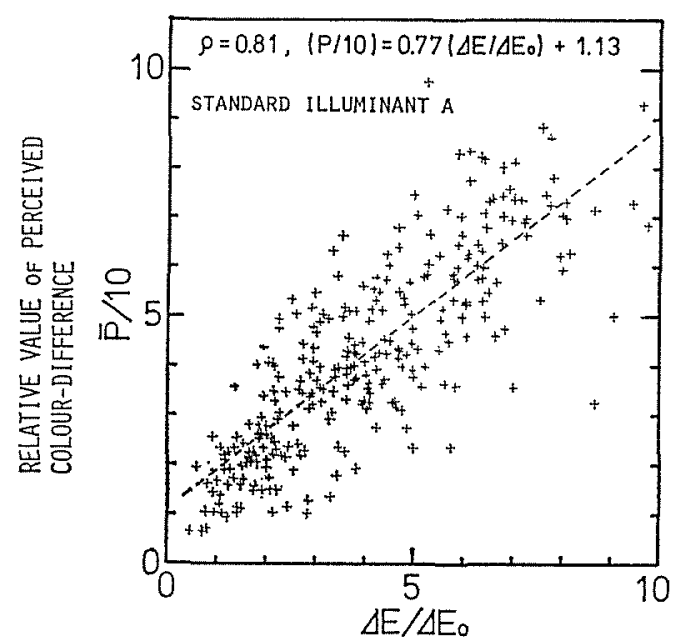

(b) CIE L*a*b* Space

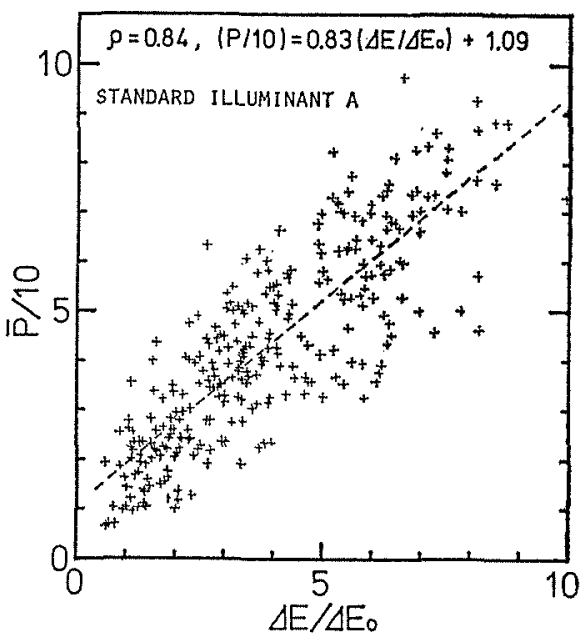

RELATIVE VALUE OF COLORIMETRIC COLOUR-DIFFERENCE

(c) Modified $L^{*} u^{*} V^{*}$ Space

(d) Modified Cube-root Space

Fig. 5 Correlation diagrams of the relative values of perceived colour-differences under the standard illuminant $A$ to colorimetric ones. 
the perceived colour-difference of one test pair, the arithmetic mean of sixteen times of judgements in the experiment $I$ and thirty times in the experiment II by two observers was taken.

The condition of the colour-difference estimation experiment under the daylight $D_{65}$ and that under the standard illuminant $\mathrm{A}$ are shown in Fig. 3 .

\section{Relations between the relative values of perceived collour - differences and those of colorimetric ones}

The value $\bar{P}$ was obtained by multiplying the ratio of the perceived colour-difference of the test pair to that of the standard one by ten. So, the relative value of perceived colour-difference is defined by $\bar{P} / 10$. On the other hand, the relative value of colorimetric colour-difference is defined by the ratio $\Delta E / \Delta E_{0}$ of the colorimetric colour-difference $\Delta E$ of the test pair to that $\Delta E_{0}$ of the standard one.

In order to examine the correlations between the colorimetric colour-differences and the perceived ones, the correlation diagrams of the relative values $(\bar{P} / 10)$ of perceived colour-differences under the daylight $\mathrm{D}_{65}$ to the relative values $\left(\Delta E / \Delta E_{0}\right)$ of colorimetric ones in the CIE $L^{*} u^{*} v^{*}$, the CIE $L^{*} a^{*} b^{*}$, the modified $L^{*} u^{*} v^{*}$ and the modified Cube-root spaces are shown in Fig. 4. Also, the correlations between these under the standard illuminant $A$ are shown in Fig. 5.

Under the daylight $D_{65}$ shown in Fig. 4, the correlation coefficient in any colorimetric space is within the range of 0.86 to 0.88 , which does not show any appreciable difference in the correlations, and does not give any noticeable difference in the distribution patterns. Since colorimetric colourdifferences and perceived ones are given in relative values, the same correlation is bound to show if the arrangements of the coordinates of colour chips in all colorimetric spaces are alike. This can be appreciated from the coordinates of the colour chips shown in Fig. 1.

On the other hand, under the standard illuminant $A$, correlation coefficients of 0.81 to 0.84 show little difference as shown in Fig. 5, just as in the case of those under the daylight $D_{65}$. The results, however, in the GIE $L^{*} u^{*} v^{*}$ and the modified $L^{*} u^{*} V^{*}$ spaces show the state of distributions dissimilar to the cases under the daylight $\mathrm{D}_{65}$. In the CIE $L^{*} u^{*} v^{*}$ space in particular, the gradient of regression line is a very low value of 0.62 . This is because the gradient tends to become extremely small in the colour chips' group which the standard colour chips are located in the vicinity of the horizontal axis $u^{*}$.

As described, with regard to the relations between the colorimetric colour-differences and perceived ones under each individual illuminant, the CIE $L^{*} u^{*} v^{*}$ space may show slightly poor relations under the standard illuminant $A$ in terms of regression coefficient, but insofar as the correlation coefficient is concerned, there is no large difference between the colorimetric spaces under any of these illuminants.

\section{Relations between perceived colour-differences and colori- metric ones when illuminant is changed}

Fig. 6 shows the correlation diagrams of the relative values of the perceived colour-differences under the standard illuminant $A$ to those under the daylight $\mathrm{D}_{65}$. The correlation coefficient is 0.95 , which is considerably high. The gradient of the regression line is 0.94 , which shows almost 1 to 1 correspondence. This shows that the relative values of the perceived colour-differences are nearly the same under the daylight $D_{65}$ and under the standard illuminant $\mathrm{A}$, and that colour-differences to be perceived change little with the change of the illuminant.

To investigate just how the relative values of perceived colour-differences vary with hue in the Munsell renotation system when the illuminant is changed from the daylight $D_{65}$ to the standard

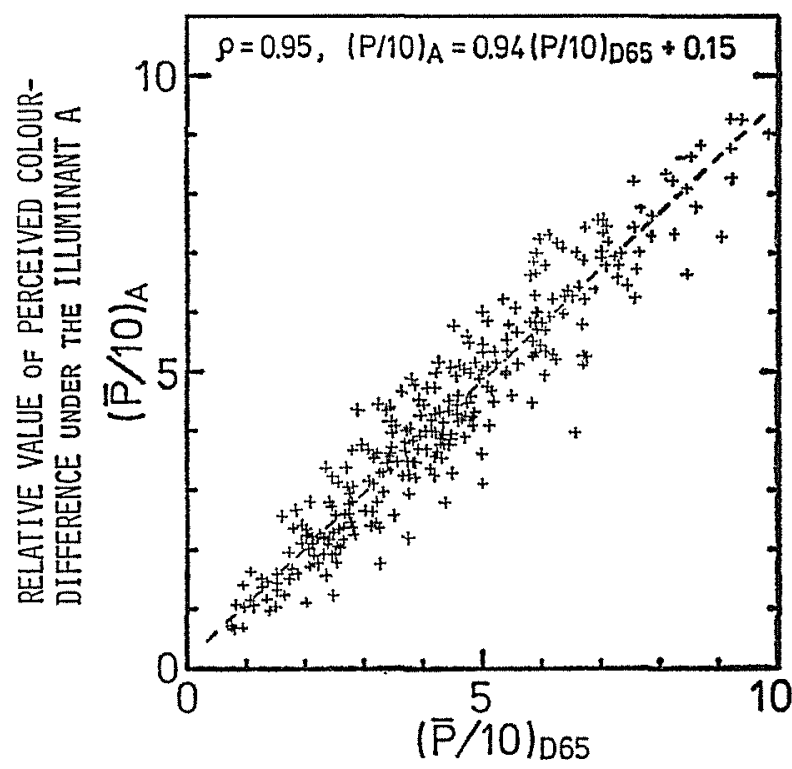

RELATIVE VALUE OF PERCEIVED COLOURDIFFERENCE UNDER THE DAYLIGHT $D_{65}$

Fig. 6 Correlation diagram of perceived colour. differences under the standard illuminant $A$ to those under the daylight $D_{65}$.

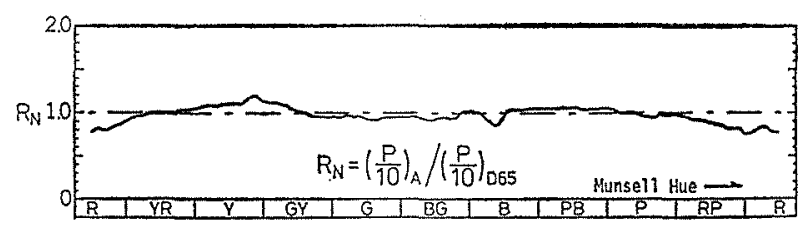

Fig. 7 Variation of the ratios of the relative values of perceived colour-differences under the standard illuminant $A$ to those under the daylight $D_{05}$ caused by hue. 
illuminant $A$, the ratio $(\bar{P} / 10) A /(\bar{P} / 10) D_{65}$ of the relative value of perceived colour-difference under the standard illuminant $A$ for each test pair to that under the daylight $D_{65}$ is calculated. The calculated ratios are averaged for all pairs which cover the same hue for the smooth curve as shown in Fig. 7 . In Fig. 7, the average value of the ratios over the entire hue range is 0.97 , and the standard deviation is 0.09 . Although the average figure is somewhat small than 1.0 , the relative values of the perceived colour-differences under the standard illuminant $A$ tend to be greater than those under the daylight $D_{65}$ near $Y$ on the hue axis.

Correlations between the relative values of the colorimetric colour-differences under the standard illuminant $A$ and those under the daylight $D_{65}$ in the colorimetric spaces are shown in Fig. 8 . The modified Cube-root, the modified $L^{*} u^{*} v^{*}$, and the CIE $L^{*} a^{*} b^{*}$ spaces have the correlation coefficients in excess of 0.95 . Also, the gradients of regression lines come close to 1.0 , and the lines go past near the origin of the coordinate axes. But in the CIE $L^{*} u^{*} v^{*}$ space, the correlation coefficient is as low as 0.77 , and the gradient is 1.06 .

This is because the correction for chromatic adaptation has been made on the basis of the von Kries law in the former three colour spaces, but in the CIE $L^{*} u^{*} v^{*}$ space a correction has been made merely by setting the coordinates of the illuminants on the origin.

To investigate just how the colorimetric colourdifferences vary with the Munsell hue when the illuminat is changed from $D_{6 \breve{5}}$ to $A$, the ratio $\left(\Delta E_{A} / \Delta E_{D 65}\right)$ of the colorimetric colour-difference
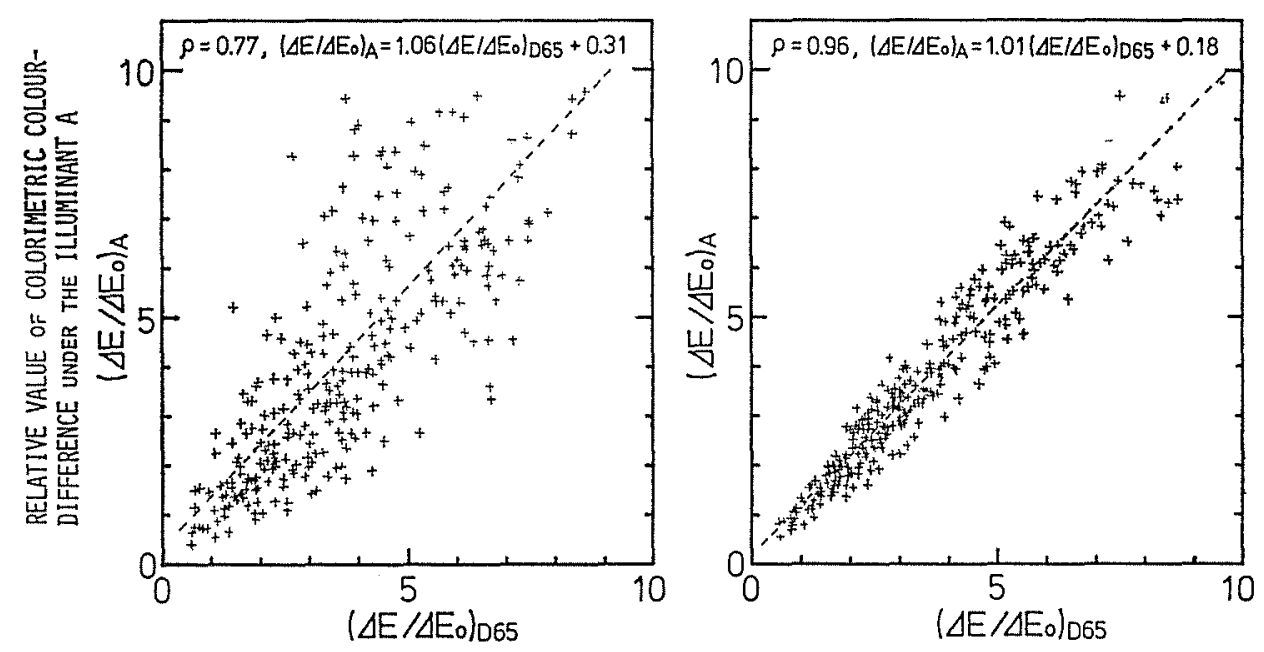

RELATIVE VALUE OF COLORIMETRIC COLOUR-DIFFERENCE UNDER THE DAYLIGHT $D_{65}$

(a) CIE L*u*v* Space

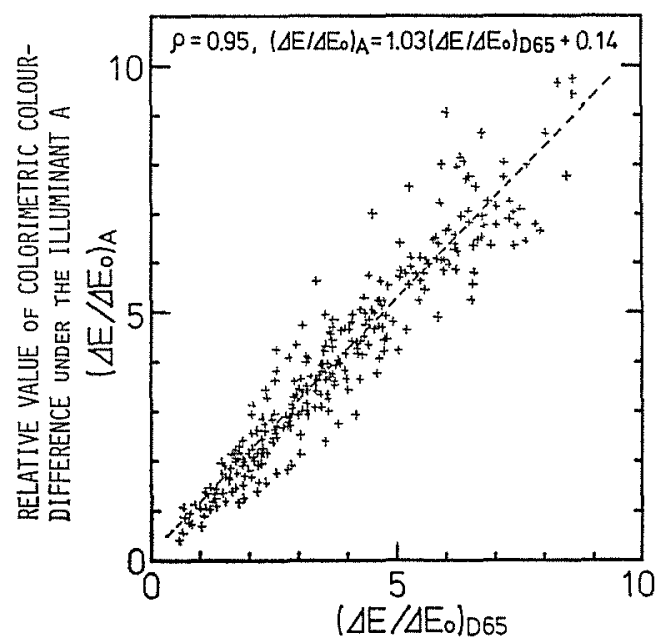

(b) CIE L*a*b* Space

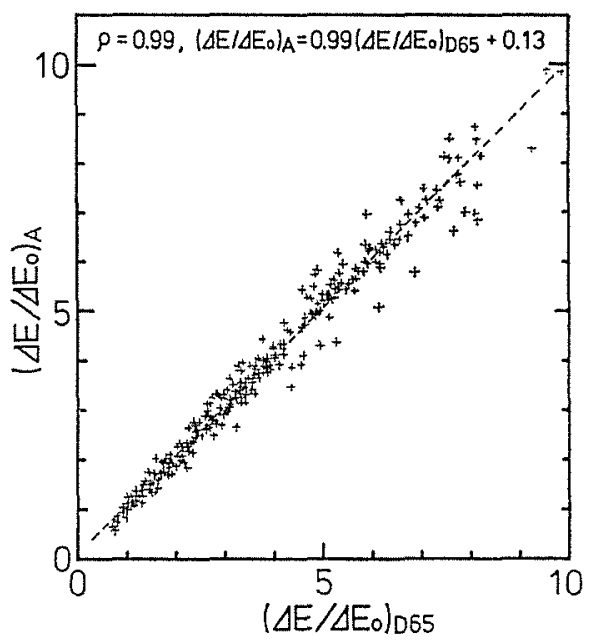

RELATIVE VALUE OF COLORIMETRIC COLOUR-DIFFERENCE UNDER THE DAYLIGHT $D_{65}$

(c) Modified $L^{*} u^{*} V^{*}$ Space (d) Modified Cube-root Space

Fig. 8 Correlation diagrams of the relative values of colorimetric colour-differences under the standard illuminant $A$ to those under the daylight $D_{65}$. 

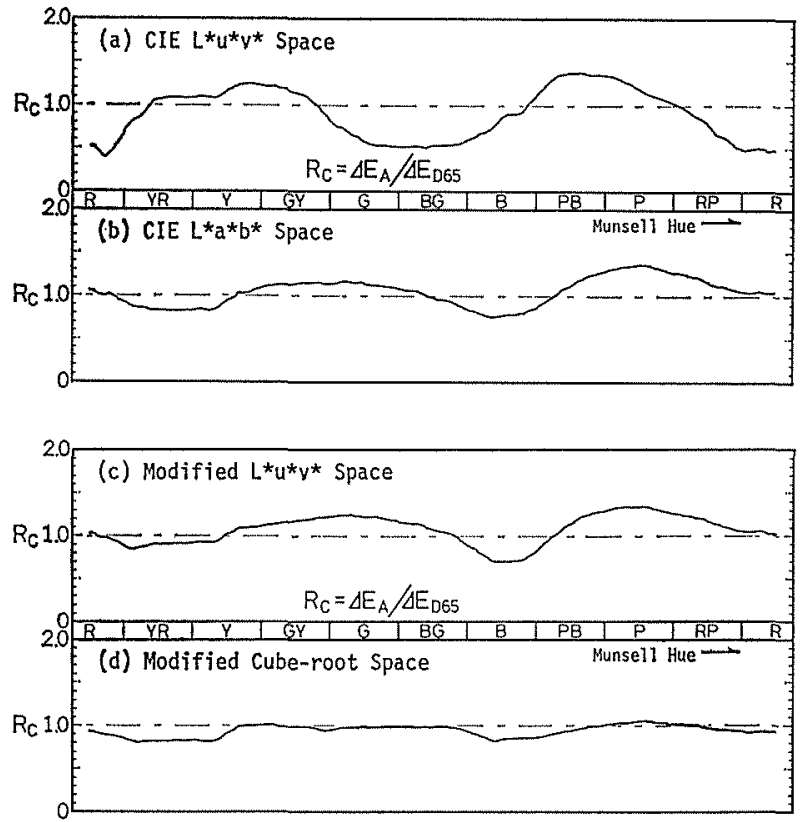

Fig. 9 Variations of the ratios of colorimetric colourdifferences under the standard illuminant $A$ to those under the daylight $D_{05}$ caused dy hue.

under the standard illuminant $A$ for each test pair to that under the daylight $D_{65}$ is graphically represented in Fig. 9 in the same manner as that in Fig. 7. In the CIE $L^{*} a^{*} b^{*}$, the modified $L^{*} u^{*} v^{*}$, and the modified Cube-root spaces, the values of $\triangle E A / \triangle E D_{65}$ are less than 1.0 near $\mathrm{YR}$ on the hue axis and $B$, and these are greater than 1.0 in the vicinity of $G$ and $P$. Also, the values are smaller at $B G$ and $R$, and larger near $P B$ and $Y$ in the CIE $L^{*} u^{*} v^{*}$ space, thus indicating the greatest variation width of 0.4 time to 1.38 times in the four spaces. When these are compared with those of the perceived colour-differences shown in Fig. 7, it can be considered that the variation of perceived colour-differences by the change of the illuminant and that of colorimetric ones in the Cube-root space show relatively good correspondency with each other.

By defining the ratio of the relative value of perceived colour-difference to that of colorimetric one, that is, $R=(\bar{P} / 10) /\left(\Delta E / \Delta E_{0}\right)$ as the perceived colour-difference per unit colorimetric one, the correlations between this value under the standard illuminant $A$ and that under the daylight $D_{65}$ are shown in Fig. 10. This correlation indicates how the variations of perceived colour-differences by the change of the illuminant and those of colorimetric ones are related to each other. Ideally, the perceived colour-differences per unit colorimetric one under any illuminant should be constant. In Fig. 10, good relations can be established if scattered points converge on the line passed the origin and with the gradient of 1.0 , but in the CIE $L^{*} u^{*} v^{*}$ space the points are widely scattered almost without correlation. That is, the correlation coefficient is not only the low value of 0.35 , but also the gradient of the regression line is the very low value of 0.38 . In the CIE $L^{*} a^{*} b^{*}$ and the modified $L^{*} u^{*} v^{*}$ spaces, the correlation coefficients are the values of 0.75 and 0.73 , respectively, and also the gradients are the values of 0.74 and 0.78 . Further, the modified Cube-root space with the correlation coefficient of 0.81 and the gradient of 0.83 shows the highest correlation among these spaces. Therefore, it may be able to pronounce that these colorimetric spaces except the CIE $L^{*} u^{*} v^{*}$ space are very good from the standpoint of compensation for chromatic adaptation.

The modified Cube-root space has very good characteristics in point of compensation for chromatic adaptation. By reviewing the Reilly Coefficients in the equation (4), or by developing four variables $R, Y, G$, and $B$ with stimulus $Y$ introduced in addition to three stimuli $R, G$, and $B$, transformation into still better colorimetric space may possibly be made.

\section{Conclusions}

The correlation coefficients and the gradients of regression lines are listed in Table 1. Also, the values of experimental results on the low chroma colour chips so far estimated are also given in Table 1 for purposes of comparison.

The comparison and review of relations between the perceived colour-differences and the colorimetric ones in four types of colorimetric spaces are summarized:

(1) Results on high chroma colour chips show

Tabel 1 Correlation Coefficients $(\rho)$ and Gradients $(\alpha)$ of Regression Lines (Values in parenthesis denote those of low chroma colour chips)

\begin{tabular}{|c|c|c|c|c|c|}
\hline & & $\operatorname{CIE} L^{*} u^{*} v^{*}$ & $\operatorname{CIE} L^{*} a^{*} b^{*}$ & Modified $L^{*} b^{*} v^{*}$ & Modified Cube-root \\
\hline \multicolumn{2}{|l|}{$\bar{P}_{A} / \bar{P}_{D 65}$} & \multicolumn{4}{|c|}{$\begin{array}{l}\rho=0.95(0.97) \\
\alpha=0.94(1.02)\end{array}$} \\
\hline \multicolumn{2}{|c|}{$\left(\frac{\Delta E}{\Delta E_{0}}\right)_{A} /\left(\frac{\Delta E}{\Delta E_{0}}\right)_{D_{65}}$} & $\begin{array}{l}\rho=0.77(0.71) \\
\alpha=1.06(0.92)\end{array}$ & $\begin{array}{l}\rho=0.96(0.90) \\
\alpha=1.01(0.90)\end{array}$ & $\begin{array}{l}\rho=0.95(0.91) \\
\alpha=1.03(1.04)\end{array}$ & $\begin{array}{l}\rho=0.99(0.98) \\
\alpha=0.99(1.04)\end{array}$ \\
\hline \multirow{2}{*}{$\left(\frac{\bar{P}}{10}\right) /\left(\frac{\Delta E}{\Delta E_{0}}\right)$} & $\mathrm{D}_{6 \tilde{5}}$ & $\begin{array}{l}\rho=0.87(0.84) \\
\alpha=0.90(0.80)\end{array}$ & $\begin{array}{l}\rho=0.86(0.83) \\
\alpha=0.92(0.80)\end{array}$ & $\begin{array}{l}\rho=0.88(0.88) \\
\alpha=0.89(0.84)\end{array}$ & $\begin{array}{l}\rho=0.87(0.85) \\
\alpha=0.87(0.86)\end{array}$ \\
\hline & A & $\begin{array}{l}\rho=0.84(0.88) \\
\alpha=0.62(0.68)\end{array}$ & $\begin{array}{l}\rho=0.83(0.78) \\
\alpha=0.83(0.79)\end{array}$ & $\begin{array}{l}\rho=0.81(0.74) \\
\alpha=0.77(0.65)\end{array}$ & $\begin{array}{l}\rho=0.81(0.78) \\
\alpha=0.83(0.78)\end{array}$ \\
\hline \multicolumn{2}{|l|}{$R_{A} / R_{D 65}$} & $\begin{array}{l}\rho=0.35(0.03) \\
\alpha=0.38(0.03)\end{array}$ & $\begin{array}{l}\rho=0.75(0.76) \\
\alpha=0.74(0.87)\end{array}$ & $\begin{array}{l}\rho=0.73(0.77) \\
\alpha=0.78(1.25)\end{array}$ & $\begin{array}{l}\rho=0.81(0.92) \\
\alpha=0.83(1.12)\end{array}$ \\
\hline
\end{tabular}



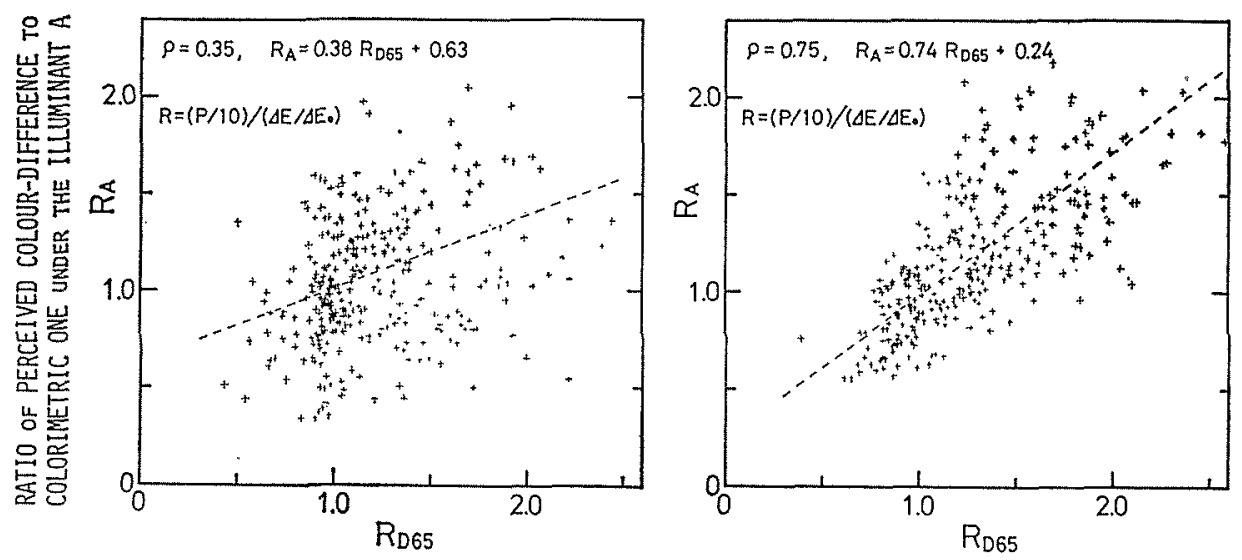

RATIO OF PERCEIVED COLOUR-DIFFERENCE TO COLORIMETRIC ONE UNDER THE DAYLIGHT $\mathrm{D}_{65}$

(a) CIE L* $u^{*} v^{*}$ Space

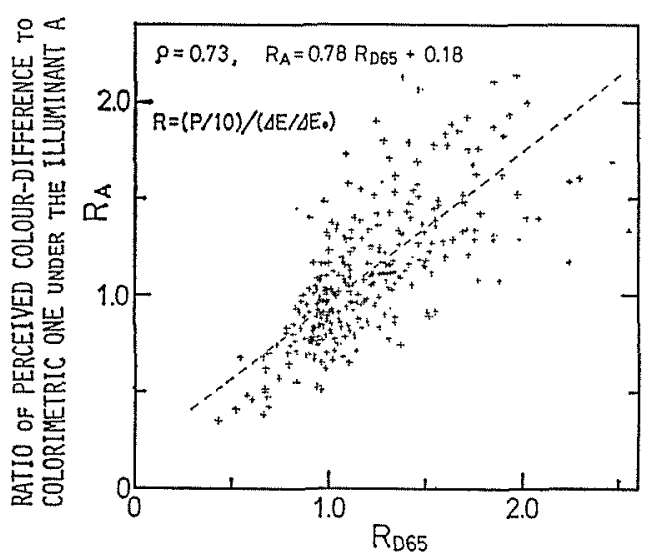

(b) CIE L*a*b* Space

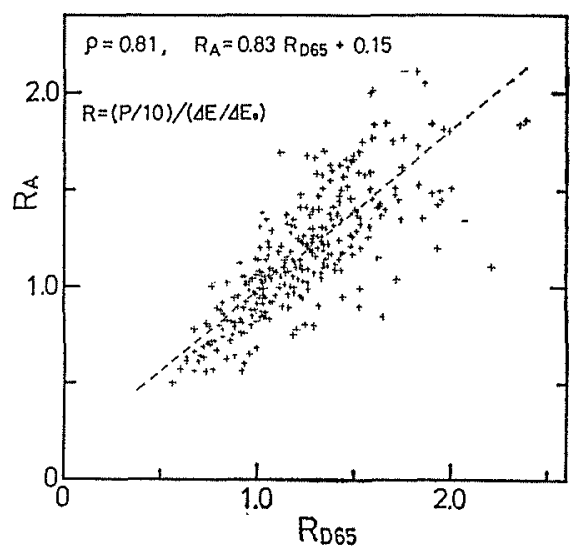

RATIO OF PERCEIVED COLOUR-DIFFERENCE TO COLORIMETRIC ONE UNDER THE DAYLIGHT $\mathrm{D}_{65}$

(c) Modified $L^{*} u^{*} v^{*}$ Space (d) Modified Cube-root Space

Fig. 10 Correlation diagrams of perceived colour-differences per unit colorimetric colourdifference under the standard illuminant $A$ to those under the daylight $D_{05}$.

that in general there is no large difference from those on low chroma colour chips.

(2) The relative values of perceived colour-differences change little with the change of the illuminant, and the constancy of perceived colour-difference holds.

(3) As the correlation between perceived colourdifferences and colorimetric ones, the CIE $L^{*} u^{*} v^{*}$ space under each individual illuminant is almost the same as other spaces under the same condition, but when the illuminant is changed, the correction corresponding to the chromatic adaptation of the visual system does not suffice.

(4). In two uniform colour spaces currently under recommendation by the CIE, the CIE $L^{*} a^{*} b^{*}$ space is better at representing the perceived colourdifferences from the standpoint of compensation for chromatic adaptation when the illuminant is changed.

(5) The modified $L^{*} u^{*} v^{*}$ space improves the CIE $L^{*} u^{*} v^{*}$ space from the standpoint of compen- sation for chromatic adaptation.

(6) The modified Cube-root space shows good characteristics from the standpoint of compensation for chromatic adaptation. Much improvement can be expected by modifying the Reilly coefficients in such a way that the perceived colour-differences can be better correlated under the daylight $D_{65}$.

(7) Under each individual illuminant, it is hard to say just which space is particularly good. This points up the need for developing a further improved space.

The relations between the perceived colour-differences and colorimetric ones in four types of colorimetric spaces studied in this investigation show little difference under each individual illuminant, but when the illuminant is changed, the relations will be better in the spaces considered compensation for chromatic adaptation. This indicates that the von Kries Coefficient Law is effective.

Colour-differences have been studied so far with equal Value $\mathrm{V}=6$ and equal Chroma $\mathrm{C}=2$ to 4 and 
$\mathrm{C}=8$; however, there is the need for further investigating the colour-differences over a broad range of lightness, chroma, and hue.

\section{References}

(1) "Official Recommendations on Uniform Colour Spaces, Colour-Difference Equations, and Metric Colour Terms", Supplement No. 2 to CIE Publication No. 15, Colorimetry (E-1.3.1) 1971, (1976).

(2) "Guidelines for Coordinated Research on Colour Difference Evaluation", Prepared by the Colour Difference Subcomm. ittee of CIE TC-1.3 (Colorimetry), (1978).

(3) Ikeda, K., Nakayama, M. and Obara, K.: Papers of Techn ical Meeting on VISUAL INFORMATION, The Institute of Electrical Engineers of Japan (IEE of Japan), VIN-77-
9 (1977) 9-1 (in Japanese).

(4) Ikeda, K., Nakayama, M. and Obara, K.: Journal of the Color Science Association Jof Japan (J. Color Sci. Assoc. Jpn), 3-1 (1978) 12 (in Japanese).

(5) Ikeda, K., Nakayama, M. and Obara, K.: CIE Publication No. 50 (1980) 83

(6) Ikeda, K., Nakayama, M. and Obara, K. : Papers of Technical Meeting on LIGHT APPLICATION AND VISUAL. SYSTEM, IEE of Japan, LAV-80-22 (1980) 42 (in Japanese).

(7) Komatubara, H., Hirai, T., Sima, Y. and Naganuma, E.: J. Color Sci. Assoc. Jpn., 5-1 (1981) 28 (in Japanese).

(8) Wyszecki, G.: J. Opt. Soc. Am., 58-2 (1968) 290

(9) Ikeda, K., Nakayama, M. and Obara, K.: Acta Chromatica. 3-2 (1977) 64 .

Received 25 Mar. 1982; Revision Received 14 May. 1982, 